\title{
Easing Infertility Problems Using Hysterosalpingography And Hysteroscopy In Al-Khoms Hospital, Libya
}

\author{
Mahmoud M. Osela \\ The Head, Dept. Of Obstetrics And Gynaecology, Faculty Of Medicine, Al-Margeb University, Al-Khoms, Libya
}

\begin{abstract}
Infertility is estimated to affect as many as 186 million people worldwide. Although male infertility contributes to more than half of all cases of global childlessness, infertility remains a woman's social burden. Fertility rate is declining in Arab world including Libya. This study is framed to overcome the infertility problem like tubal, uterine and cervical abnormalities among Libyan women by using assisted reproductive techniques (ART) like Histerospingography and Histeroscopy and to find out the success rate of this technology among Libyan population. A Study has conducted in Department of Obstetrics and Gynecology, Al-khoms teaching Hospital, Libya for 1 year during 2015 with 144 cases who had previous surgery history between the age group of 18 and 45 years. Study of infertility problem was diagnosed and treated by Hysterosalpingography (HSG) and Hysteroscopy methods. HSG was performed first followed by hysteroscopy. Results showed that more (20) cases (13.89\%) were with kinked tube and peritubal adhesion and 15 cases (10.42\%) were with Hang up tubes and open problems. Concluded that HSG in combination with Hysteroscopy, assisted reproductive technology is easy methodology with less or without anaesthetic hazards and free of surgical complications. After the detection and treatment, the success rate of fertility was $45 \%$ in this study.

Keywords: Infertility, Salpigitis, Histerospingography and Hysteroscopy.
\end{abstract}

\section{Introduction}

Infertility or the inability to conceive remains a problem of global proportions. Female infertility affects estimated 48.5million women in the world with the highest prevalence of infertility affecting people in South-Asia, Sub-saharan Africa, North Africa/Middle east, Central or Eastern Europe or central Asia (Mascarenhas et al., 2012). According to American Society for Reproductive medicine (ASRM, 2013), Age, Smoking, Sexually Transmitted Infections and Being Overweight or Underweight can all affect fertility. Infertility was also found to be caused by disorders in both the male and female partners together; thus in $39 \%$ of cases both the man and woman presented with disorders. The woman alone was responsible for infertility in one-third of cases and the man alone in one-fifth. Unexplained infertility was found in $8 \%$ of the couples surveyed (Thonneau et al., 1991).

Common causes of infertility include male factor (45\%), ovulation disorders (37\%) and tubal damage (18\%) (Farhi and Ben-Haroush, 2011). A combination of several factors is found in approximately $20 \%$ of all couples. The etiology of tubal damage can be intrinsic (ascending salpingitis, including salpingitis isthmica nodosa) or extrinsic (peritonitis, endometriosis and pelvic surgery). Salpingitis is the most common serious infection in women of reproductive age. Polycystic ovarian syndrome (PCOS) is another most common gynecological endocrinopathy (Kauffman et al., 2008). PCOS appears to be associated with an increased risk of metabolic aberrations, including insulin resistance and hyperinsulinism, type 2 diabetes mellitus, dyslipidemia, cardiovascular disease, and endometrial carcinoma (Legro, 2003). Uterine abnormalities might have a causal role in infertility and in the recurrent miscarriages whether of the first or second trimester (Propst and Hill, 2000). Uterine abnormalities perhaps impair the proper embryo implantation and growth due to poor vascularisation (Brown et al., 2000). Basic investigations for female infertility include assessment of cervical, uterine, tubal and ovulatory factors. Traditionally, uterine shape and fallopian tubes were assessed by Hysterosalpingogram (HSG). HSG is a special fluoroscopic x-ray procedure to evaluate the patency (openness) of the fallopian tubes and the shape of the uterine cavity. However, in the recent past, hysteroscopy is being increasingly used for direct visualization of uterine cavity and is considered superior to HSG. Hysteroscopy is used to diagnose or treat problems of the uterus (kumar et al., 2003). Aim of this study is to detect the infertility problem like tubal, uterine and cervical abnormalities and to find out the suitability of HSG and Hysteroscopy (assisted reproductive technology) in easing the infertility problems among Libyan women presented in Alkhoms teaching hospital, Libya.

\section{Materials And Methods}

A Study was conducted in Department of Obstetrics and Gynecology, Al-khoms teaching Hospital, Libya for one year during 2015 with 144 cases (age group of 18 and 45 years). Cases involved in this study were with the previous surgery history and complain of infertility even after 3 months of ovulation induction and no 
pregnancy in spite of normal semen parameters. This study was aimed to detect and cure the uterine, tubal and cervical abnormalities of the women who had infertility problems. Previous surgery history data has collected by the Doctors in the same ward. In this Study, infertility problem was diagnosed and treated by Hysterosalpingography (HSG) and Hysteroscopy methods. HSG was performed first followed by hysteroscopy.

Hysterosalpingography: Hysterosalpingography was performed in the proliferative phase of menstrual cycle without using analgesics or anaesthesia. Half an hour before HSG, injection Buscopan (Hyoscin Butyl Bromide) $0.5 \mathrm{ml}$ was given intramuscularly to avoid tubal spasm. Cervix was visualized with the help of Sim's speculum and the anterior lip of cervix was held with tenaculum. Water-soluble contrast media was injected slowly by using Leech Wilkinson canula and the films were taken under fluoroscopic monitoring. This study was performed in the Hysterosalpingogram, Model-PS.60L/6, Toshiba Corporation, Japan.

Hysteroscopy: Hysteroscopy was performed under general anaesthesia in the proliferative phase of the menstrual cycle. Cervical dilatation was performed (when necessary) to enable the insertion of the hysteroscope. Glycine $1.5 \%$ was used as distension medium. Operative hysteroscopy was performed to remove the discovered anomalies. Hysteroscopic findings were documented.

Then follow up of the cases were done continuously to find out the successive rate of pregnancy among the cases undergone in this study.

\section{Results And Discussion}

Hysterosalpingography is a useful screening test for the evaluation of the uterine cavity. If a hysterogram demonstrates intrauterine abnormalities, hysteroscopy should be considered to make a definite diagnosis and treatment. Both procedures should be complementary to each other (Preutthipan and Linasmita, 2003). This study was aimed to detect and cure the uterine, tubal and cervical abnormalities of the women who had infertility problems. The primary role of HSG is to evaluate the morphology and the patency of the fallopian tubes. HSG can also be helpful in evaluating uterine cavity abnormalities. It is considered to have a high sensitivity (60-98\%) but low specificity (15-80\%) in detecting uterine abnormalities, and hysteroscopy remains the method of choice for the final assessment (Pundir and El Toukhy, 2010).

Cases involved in this study with previous surgical history are reported in the Table 1. It reveals that more number of cases (115) with Caesarean section (79.86\%) was observed. Other cases like Appendectomy (6.25\%), Myomectomy (4.86\%), Ectopic pregnancy (2.79\%), Cystectomy (2.08\%) and Post DNC perforation $(2.08 \%)$. Only once case $(0.69 \%)$ with Meteroplasty also involved in the study.

Table 1: Cases with previous history of surgery.

\begin{tabular}{|c|l|c|c|}
\hline S. No. & \multicolumn{1}{|c|}{ Surgery type } & No. Of cases & \% \\
\hline 1 & Appendectomy & 09 & 6.25 \\
\hline 2 & Caesarean section & 115 & 79.86 \\
\hline 3 & Cystectomy & 03 & 2.08 \\
\hline 4 & Ectopic pregnancy & 04 & 2.79 \\
\hline 5 & Hysterotomy & 02 & 1.39 \\
\hline 6 & Meteroplasty & 01 & 0.69 \\
\hline 7 & Myomectomy & 07 & 4.86 \\
\hline 8 & Post DNC perforation & 03 & 2.08 \\
\hline & Total & $\mathbf{1 4 4}$ & $\mathbf{1 0 0 . 0 0}$ \\
\hline
\end{tabular}

Table 2: Result of cases from the study.

\begin{tabular}{|c|l|c|c|}
\hline S. No. & \multicolumn{1}{|c|}{ Cases } & No. & \% \\
\hline 1 & Normal & 75 & 52.08 \\
\hline 2 & Hydrosalpinx & 06 & 4.17 \\
\hline 3 & Kinked tube and peritubal adhesion & 20 & 13.89 \\
\hline 4 & Hang up tubes and open & 15 & 10.42 \\
\hline 5 & Blocked tube & 11 & 7.64 \\
\hline 6 & Regular outline of uterine cavity & 03 & 2.08 \\
\hline 7 & Cervical polyp & 08 & 5.56 \\
\hline 8 & Endometrial polyp & 03 & 2.08 \\
\hline 9 & Closed cervix with failed HSG procedures & 03 & 2.08 \\
\hline & Total & $\mathbf{1 4 4}$ & $\mathbf{1 0 0 . 0 0}$ \\
\hline
\end{tabular}

Of the 144 HSG and Hysteroscopy examinations, 75 cases (52.08\%) were observed normal (Table 2). 20 cases $(13.89 \%)$ were with kinked tube and peritubal adhesion and 15 cases $(10.42 \%)$ were with Hang up tubes and open problems. Other complications like Blocked tubes in 11 persons $(7.64 \%)$, Cervical polyp in 8 persons $(5.56 \%)$ and Hydrosalpix in 6 persons $(4.17 \%)$ and least $2.08 \%$ were observed each with Regular outline of uterine cavity, Endometral polyp and Closed cervix with failed HSG procedures in 3 persons. From this study it was observed that HSG in combination with Hysteroscopy assisted reproductive technology is easy 
methodology with less or without anaesthetic hazards and free of surgical complications. After the detection and treatment, the success rate of fertility was $45 \%$ in this study.

Similar studies like infertility problems and its method of diagnose and treatments were by many researchers. Most uterine abnormalities were mild adhesions, small submucous myomas and polyps and their incidence was greater in women aged $\geq 30$ years and women with secondary infertility. The overall pregnancy rate in the treated women within one year of follow up was $46 \%$. As a cause of unexplained infertility, subtle uterine abnormalities are diagnosed only during hysteroscopy and they are relatively common in infertility women. Although the presence of these abnormalities is not detected by the basic investigations for infertility, their correction seems to be necessary when infertility is desired and other infertility causes are excluded (Elbareg et al., 2014).

Adrian et al., (2012) have expressed from their study that HSG is a safe, low cost and, with the application of propofol sedation, a well-tolerated procedure for tubal assessment, which should be performed at the end of the infertility investigation protocol. The relatively low percentage $(21 \%)$ of pathological exams in our population underlines the need for good patient preselection. The high false-positive rate for proximal tubal occlusion (39\%), probably due to tubal spasm, demonstrates the importance of antiperistaltic agents and delayed imaging. Furthermore, we observed a high pregnancy rate (in patients with pathological HSG). Most cases of pregnancy were spontaneous without tubal or uterine surgery. This could mean that the indication for HSG was too broad or that there could even be a therapeutic effect of the HSG procedure, i.e., improved patency of the fallopian tube because of the flushing during the examination (Johnson et al., 2005).

Abdellah et al., (2010) also explained that Hysteroscopic evaluation of the uterine cavity is highly valuable for women scheduled for repeated intracytoplasmic sperm injection (ICSI) procedure. Hysteroscopic correction of uterine anatomical abnormalities not only increases the success rate of ICSI but also, increases the spontaneous pregnancy rate. We recommend the IVF/ embryo transfer centres to establish diagnostic hysteroscopy as an essential preliminary step for patients scheduled for repeat ICSI procedures.

\section{Conclusion}

HSG in combination with Hysteroscopy assisted reproductive technology is easy methodology with less or without anaesthetic hazards and free of surgical complications. After the detection and treatment, the success rate of fertility was $45 \%$ in this study. The effectiveness of fertility treatment is primarily dependant on three factors, namely the woman's age, the duration of infertility and whether the woman has been previously pregnant. These factors are, in most circumstances, more important than the actual clinical diagnosis and determine not only the outcome of treatment, but also the appropriateness and timing of intervention. Furthermore lifestyle, particularly obesity and smoking, is increasingly relevant, and can affect both fertility and the outcome of treatment.

\section{References}

[1]. Abdellah AH, Rasheed SM and Abdelraheem M (2010) Hysteroscopic evaluation of the uterine cavity after Intracytoplasmic sperm injection and embryo transfer failure: Is it rewarding? Abstracts of the 17th Annual Meeting. Middle East Fertility Society Damascus, Syria 6 to 9 October 2010.

[2]. Adrian CS, Nikola F, Cornelia U, Mikael KH and Rahel AK (2012) Hysterosalpingography in the workup of female infertility: indications, technique and diagnostic findings. Insights Imaging 3(5):476-483.

[3]. ASRM (2013), Fertility: assessment and treatment for people with fertility problems. NICE clinical guideline CG156.

[4]. Brown SE, Coddington CC, Schnorr J, Toner JP, Gibbons W, Oehninger S (2000) Evaluation of outpatient hysteroscopy, saline infusion hysterosonography, and hysterosalpingography in infertile women: a prospective, randomized study. Fertil Steril.74: 10291034.

[5]. Farhi J and Ben-Haroush A (2011) Distribution of causes of infertility in patients attending primary fertility clinics in Israel. Isr Med Assoc J. 13(1):51-55.

[6]. Elbareg AM, Essadi FM, Anwar KI and Elmehashi (2014) Value of hysteroscopy in management of unexplained infertility. Asian Pacific J of reproduction 3(4):295-298.

[7]. Johnson N, Vandekerckhove P, Watson A, Lilford R, Harada T and Hughes E (2005) Tubal flushing for subfertility.Cochrane Database Syst Rev. 18(2):CD003718.

[8]. Kauffman RP, Bake TE, Baker VM, DiMarino P and Castracane VD (2008) Endocrine and metabolic differences among phenotypic expressions of polycystic ovary syndrome according to the 2003 Rotterdam consensus criteria. American Journal of Obstetrics \& Gyne- cology 198:e1-670.e10.

[9]. Kumar S, Aswathi RT and Gokhale N (2003) Assessment of uterine factor in infertile women: Hysterosalpingography vs Hysteroscopy. MJAFI 60(1):39-41.

[10]. Legro RS (2003) polycystic ovary syndrome and car- diovascular disease: A premature association? Endocrine Reviews 24:302-312

[11]. Mascarenhas M.N., Flaxman S.R., Boerma T., Vanderpoel S., Stevens G.A. (2012). National, Regional, and Global Trends in Infertility Prevalence Since 1990: A Systematic Analysis of 277 Health Surveys. PLOS Med 9 (12): e1001356.

[12]. Preutthipan S and Linasmita V (2003) A prospective comparative study between hysterosalpingography and hysteroscopy in the detection of intrauterine pathology in patients with infertility. The J Obst. and Gynec. Research 29(1):33-37.

[13]. Propst AM and Hill JA (2000) Anatomic factors associated with recurrent pregnancy loss. Semin Reprod Med 18: 341-350.

[14]. Pundir J, El Toukhy T (2010) Uterine cavity assessment prior to IVF. Womens Health (Lond Engl) 6(6):841-848.

[15]. Thonneau P, Marchand S, Tallec A, Ferial M, Ducot B, Lansac J, Lopes P, Tabaste J and Spira A (1991) Incidence and main causes of infertility in a resident population (1 850 000) of three French regions (1988-1989) Human Reproduction 6(6):811-816. 